\title{
Class field theory, its three main generalisations, and applications
}

\author{
Ivan Fesenko
}

\begin{abstract}
This work presents branches of class field theory. Special and general approaches to class field theory, and their roles, are discussed. Three main generalisations of class field theory: higher class field theory, Langlands correspondences and anabelian geometry, and their further developments are discussed. Several directions of unification of generalisations of class field theory are proposed. New fundamental open problems are included.
\end{abstract}

Class Field Theory (CFT) is the main achievement of algebraic number theory of the 20th century. Its reach, beauty and power, stemming from the first steps in algebraic number theory by Gauß, have substantially influenced number theory. Shafarevich wrote [12, Foreword]:

Weil was undoubtedly right when he asserted, in the preface to the Russian edition of his book on number theory [72], that since class field theory pertains to the foundation of mathematics, every mathematician should be as familiar with it as with Galois theory. Moreover, just like Galois theory before it, class field theory was reputed to be very complicated and accessible only to experts. [...] For class field theory, on the other hand, there is a wide range of essentially different expositions, so that it is not immediately obvious even whether the subject is the same.

Weil's opinion has proved to be quixotic: these days even some number theorists are not familiar with the substance of CFT.

This text reviews the enduring process of discovering new branches of CFT and its generalisations. Many of such developments were complicated at their early stages and some were difficult or impossible to understand for their contemporaries. Three main generalisations of CFT and their further extensions will be presented and some of their key fundamental features will be discussed. This text proposes eight fundamental problems.

We start with Kummer theory, a purely algebraic exercise, whose highly nontrivial arithmetic analogues over arithmetic fields are supplied by CFT. Kummer theory is

2020 Mathematics Subject Classification. 11-XX, 11R37, 11S37, 12-03, 19 F05.

Keywords. Class field theory, general class field theory, special class field theory, higher class field theory, Langlands correspondences, anabelian geometry, elliptic curves over global fields, zeta integrals, higher adelic geometry and analysis, IUT theory. 
an algebraic predecessor of CFT including its existence theorem. Then we discuss the fundamental split of (one-dimensional) CFT into special CFT (SCFT) and general CFT (GCFT). This split has enormously affected many developments in number theory. Section 3 delves into four fundamental parts of CFT including the reciprocity map, existence theorem, explicit formulas for the Hilbert symbol and its generalisations, and interaction with ramification theory. Section 4 briefly touches on higher Kummer theory using Milnor $K$-groups, i.e. the norm residue isomorphism property.

Three generalisations of CFT: Langlands correspondences (LC), higher CFT, and anabelian geometry are discussed in Section 5. We note that the split of CFT into SCFT and GCFT is currently somehow reproduced at the level of generalisations of CFT: LC over number fields does not yet have any development parallel to GCFT, while higher CFT is parallel to GCFT and it does not have substantial developments similar to SCFT.

In the last section we specialise to elliptic curves over global fields, as an illustration. There we consider two further developments: Mochizuki's inter-universal Teichmüller theory (IUT) which is pivoted on anabelian geometry and two-dimensional adelic analysis and geometry which uses structures of two-dimensional CFT. We also consider the fundamental role of zeta integrals which may unite different generalisations of CFT. Similarly to the situation with LC, the current studies of special values of zeta- and $L$-functions of elliptic curves over number fields, except two-dimensional adelic analysis and geometry, use special structures and are not of general type.

There is no attempt to mention all the main results in CFT and all of its generalisations or all of their parts, and the text does not include all of bibliographical references.

\section{Kummer theory}

For a field $k$ denote its absolute Galois group by $G_{k}$ and its maximal abelian quotient by

$$
G_{k}^{\mathrm{ab}}=G_{k} /\left[G_{k}, G_{k}\right] .
$$

For a positive integer $m$ the group of roots of unity of order $m$ is denoted by $\mu_{m}$.

For a field $k$ whose characteristic does not divide integer $m>1$, the exact sequence of $G_{k}$-modules

$$
1 \longrightarrow \mu_{m} \longrightarrow \mathrm{k}^{\times} \longrightarrow \mathrm{k}^{\times} \longrightarrow 1,
$$

where $\mathrm{k}$ is a separable closure of $k$, gives the Kummer map

$$
k^{\times} / k^{\times m} \simeq H^{1}\left(G_{k}, \mu_{m}\right) .
$$

This is an $H^{1}$-theory.

If $\mu_{m}$ is in $k$, then the R.H.S. $=\operatorname{Hom}\left(G_{k}, \mathbb{Z} / m \mathbb{Z}\right)$, and we get the Kummer pairing

$$
k^{\times} / k^{\times m} \times G_{k} /\left(\left[G_{k}, G_{k}\right] G_{k}^{m}\right) \longrightarrow \mathbb{Z} / m \mathbb{Z} .
$$

Kummer duality is easily deduced: finite abelian extensions of $k$ of exponent $m$ are in explicit one-to-one correspondence with subgroups $B$ of $k^{\times}$of exponent $m: B \longrightarrow k(\sqrt[m]{B})$. The analogue of this property in CFT is existence theorem of CFT; see Section 3. 
This theory is not of much value for arithmetic fields, e.g. $\mathbb{Q}$ and $\mathbb{Q} p$ and their finite extensions, since they contain too few roots, unlike function fields over algebraically closed fields. One can slightly extend Kummer theory, working with $\mathbb{Q}\left(\mu_{m}\right)$, for prime $m$, using the fact that $\left|\mathbb{Q}\left(\mu_{m}\right): \mathbb{Q}\right|$ is prime to $m$, but this does not go too far.

First results in anabelian geometry show that every continuous automorphism of $G_{\mathbb{Q}}$ is inner. For nonarchimedean completions $k_{v}$ of number fields not every continuous automorphism of the absolute Galois group $G_{k_{v}}$ is inner. We do know an algebraic description of the absolute Galois groups of $k_{v}$ (Demushkin, Yakovlev, Jannsen-Winberg), but relations of this description with higher ramification filtration are in need of further work. For number fields we still know little about $G_{k}$, unlike $G_{k}^{\text {ab }}$ due to CFT. The Shafarevich conjecture: $\left[G_{\mathbb{Q}}, G_{\mathbb{Q}}\right]$ is free in the category of profinite groups, remains open.

Key Fundamental Problem. How to reach to the maximal separable extension, maximal abelian extension, maximal nilpotent extension of fixed nilpotent class of arithmetic fields, and how to describe their finite subextensions in terms of objects associated to the ground field and in ways which allow a good range of applications? Which structures of arithmetic fields to use for such descriptions?

This problem for abelian extensions is solved by various types of CFT and by higher CFT, for nilpotent extensions of local fields it is partially solved by arithmetic non-abelian local class field theory, whereas the Langlands correspondence provides some conjectural answers using representation theory, while anabelian geometry provides very different insights into the full structure of the absolute Galois group. Various developments that use appropriate features of CFT and its three generalisations may lead to a new powerful theory.

\section{CFT of two types}

CFT includes a construction of the reciprocity map, a homomorphism from an appropriate group associated to the ground field, to the Galois group of the maximal abelian extension of the field, and existence theorem which associates in 1-1 fashion, a finite abelian extension of the ground field to an appropriate open subgroup of the appropriate group: this open subgroup is the norm group of the abelian extension. Thus, the transfer/norm map plays the key role in $\mathrm{CFT}^{1}$, and one of key features of general CFT (see below) is its functoriality with respect to finite separable extensions of the fields.

It is of fundamental importance to distinguish two different types of CFT:

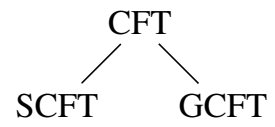

\footnotetext{
${ }^{1}$ as well as in motivic cohomologies
} 
Type I: $\boldsymbol{S C F T}=$ special $\boldsymbol{C F T}$. These diverse theories use torsion/division points or values of appropriate functions at torsion points to generate finite extensions of the base fields under investigation and to describe the Galois action on them. Historically, this is the first approach. The global number fields versions of these theories work over certain small fields only, hence they are not functorial. The local (Lubin-Tate) and functional case theories work over any field.

The list of SCFTs includes (see [19] and other historical reviews of CFT for more information):

Cyclotomic (historically, the first): Kronecker, Weber, Hilbert.

Using elliptic curves with CM (historically, the second): Kronecker (including his Jugendtraum), Weber, a relevant portion of Takagi's work.

Using abelian varieties with CM: Shimura. These theories are not extendable to arbitrary number fields.

Positive characteristic: Hayes and Drinfeld (rank 1 Drinfeld modules). These theories work over all global fields of positive characteristic.

Local theory using Lubin-Tate formal groups. It works over any local field with finite residue field and does not work over local fields with infinite perfect residue field.

Some of the SCFT are not easy. Weber published several erroneous proofs of the complex multiplication CFT, due to mistakes with the 2-primary part; see [64]. In comparison to simplified explicit versions of GCFT, SCFT perhaps stays too close to detailed structures and is not able to see more general easier to use structures. Hilbert Problem 12 was about extensions of SCFT to number fields, the best was achieved by Shimura.

Type II: $\boldsymbol{G C F T}=$ general $\boldsymbol{C F T}$. These theories follow very different conceptual patterns than SCFT. They work over any global or local field. In particular, there are fundamental conceptual features of GCFT which one does not find in SCFT. Working at the level of small number fields does not uncover various general structures which make GCFT work over any finite separable extension of the ground field.

The list of GCFTs for arithmetic fields includes:

Takagi, this seems to be the first work in GCFT with his general existence theorem and its applications;

Artin reciprocity map, whose full construction uses Chebotarev's theorem;

Hasse, the use of the Brauer group in CFT, the first local CFT, local-to-global aspects;

Chevalley's invention of ideles, local-to-global, the global reciprocity map as the product of the local reciprocity maps, whose kernel contains the diagonal image of global elements.

Classical approaches to CFT are presented, among many sources, in Hasse's Klassenkörperbericht, and in Weil's and Lang's books.

Cohomological approaches: Artin-Tate's book [2] is a classical cohomological presentation, and there are many other presentations of varying degree of understanding of what is 
actually going on. All these class field theories are not especially easy to embrace. Finding explicit formulas for the Hilbert pairing and its generalisations (see Section 3 below) was one of the ways to get more explicit information about the reciprocity map.

Positive characteristic general CFTs: there are various approaches, including some parallel to the number fields case. The GSFT Kawada-Satake theory [36] that uses Witt duality for the most difficult $p$-primary part is as easy as the SCFT theory of Hayes-Drinfeld.

Explicit, post-cohomological and cohomologically-free theories, local CFT: Tate-Dwork (Dwork's Lemma proved by Tate), Hazewinkel, Neukirch, Fesenko, global CFT: Neukirch. These theories clarified and made explicit some of the key structures of CFT. These are less dependent on torsion and they do not use the Brauer group. It is amazing that the fact that the Brauer group computation is not needed for CFT is still not well known. These explicit CFT are easy and they really explain CFT. One can say that explicit GCFT, working over any global and local field, is easier than SCFT working over small number fields only. Note the very different nature of explicitness in explicit GCFT in comparison to that in SCFT. The explicit GCFT was highly useful in developing aspects of higher CFT.

\section{Four fundamental parts of CFT}

Part I. The functorial reciprocity map

$$
\Psi_{k}: C_{k} \longrightarrow G_{k}^{\mathrm{ab}}
$$

is almost a topological isomorphism, from the topological abelian group $C_{k}$ (the multiplicative group of a local field or the idele class group of a global field) associated to the ground field $k$. For a finite Galois extension $l / k$ it induces the isomorphism

$$
C_{k} / N_{l / k} C_{l} \simeq G(l / k)^{\mathrm{ab}}
$$

Part II. The existence theorem, describing finite abelian extensions of the ground field $k$ in terms of open subgroups of finite index of $C_{k}$, is important in many applications. Existence theorem is the origin of the name 'class field': open subgroups of finite index in $C_{k}$ are in one-to-one correspondence with finite abelian extensions $l / k:^{2,3}$

$$
l / k \longrightarrow N_{l / k} C_{l}
$$

The existence theorem allows to construct and study abelian extensions by operating with open subgroups of finite index.

\footnotetext{
${ }^{2}$ In various generalisations of the classical class field theory such as class field theory for local fields with quasi-finite or perfect residue field, [5], the topology associated with norm subgroups is strictly weaker than the discrete valuation topology on the multiplicative group.

${ }^{3}$ See also [13] which connects aspects of existence theorem with topological reflexivity with respect to a related explicit pairing.
} 
Part III. Explicit formulas for the (wild) Hilbert pairing, already asked for in Hilbert's Problem 9. Important for numerous applications and computations. The nature of explicitness here differs from that in explicit CFT.

Part IV. Compatibility with ramification theory is an additional arithmetic flavour of CFT. The local reciprocity map (a) makes compatible the filtration on groups of units by higher subgroups of principal units with the higher ramification groups filtration on the abelian part of the absolute Galois group. The latter filtration, on any Galois group of a local field, is due to an application of (b) Herbrand's theorem on the compatibility of the upper ramification filtration with taking quotients of Galois groups, and it satisfies (c) the Hasse-Arf theorem: higher ramification jumps of abelian extensions are integers.

Now we discuss various features of Part I and Part III of CFT.

Cohomological approaches: there are several, all are relatively well known. Cohomological CFT is an $\mathrm{H}^{2}$-theory. One of the approaches uses the Kummer map, locally

$$
\begin{aligned}
\operatorname{Hom}\left(G_{k}, \mathbb{Z} / m \mathbb{Z}\right) \times k^{\times} / k^{\times m} & \longrightarrow H^{1}\left(G_{k}, \mathbb{Z} / m \mathbb{Z}\right) \times H^{1}\left(G_{k}, \mu_{m}\right) \\
& \longrightarrow H^{2}\left(G_{k}, \mu_{m}\right)={ }_{m} \operatorname{Br}(k) \simeq \mathbb{Z} / m \mathbb{Z},
\end{aligned}
$$

which gives the map

$$
k^{\times} \longrightarrow \lim _{\longleftarrow} k^{\times} / k^{\times m} \longrightarrow G_{k}^{\mathrm{ab}} .
$$

The map $H^{1} \times H^{1} \rightarrow H^{2}$ is defined using the cup product. The cup product is nonexplicit, which implies the non-explicit nature of cohomological class field theory.

Remark. Already Hasse used information about the Brauer group to deduce the reciprocity map. However, the information about the Brauer group is of less use for existence theorem and explicit formulas. CFT is not reduced to the computation of the Brauer group and the Brauer group is not needed in explicit CFT, see above.

Fifty years ago Hasse wrote [19]:

the sharply profiled lines and individual features of this magnificent edifice seem to me to have lost somewhat of their original splendour and plasticity by the penetration of class field theory with cohomological concepts and methods.

The class formations approach was a long search for clarification of CFT in the following sense: deduce as much as possible in CFT from as few axioms as possible. Typically, the axioms are about $H^{0}$ (index of the norm group) and $H^{1}$ (Hilbert Theorem 90) for cyclic extensions. Many papers are devoted to the CFT mechanism, which is about how to deduce all the main theorems of CFT from a small number of assumptions (CFT axioms) in a purely topological group theoretical way, hence without using ring structures. Thus, from the point of view of anabelian geometry, the CFT mechanism can play a special role. ${ }^{4}$ At the same time, proving CFT axioms in all known types of CFT involves the ring structure.

\footnotetext{
${ }^{4}$ See more on this in 'Reciprocity and IUT', https://www.maths.nottingham.ac.uk/plp/pmzibf/jl.pdf.
} 
Explicit CFT provides much more information about the image in $C_{k} / N_{l / k} C_{l}$ of a Galois automorphism $\sigma$ of a finite Galois extension $l / k$, [60,61]. The Neukirch method has a very short description, and, due to its fundamental importance and its potential value for future developments, we briefly present it now. One of its key points is to work with appropriate infinite extensions to get information about finite extensions. In explicit CFT one works with appropriate free abelian pro-extensions of rank 1: the maximal unramified extension for local fields, the extension $f k / k$ for algebraic number fields $k$ where $f / \mathbb{Q}$ is the only $\widehat{\mathbb{Z}}$-subextension of $\mathbb{Q}^{\mathrm{ab}} / \mathbb{Q}$, and the maximal constant extension of a global field of positive characteristic.

Start with an abelian (discrete topological) group $A$ endowed with a continuous action by a profinite group $G$. For simplicity, let $G$ be the absolute Galois group $G_{k}$ of a field $k$. For an open subgroup $G_{K}$ of $G_{k}$ denote by $A_{K}$ the $G_{K}$-fixed elements of $A$. For an open subgroup $G_{L}$ of an open subgroup $G_{K}$ of $G_{k}$ denote by $N_{L / K}: A_{L} \rightarrow A_{K}$ the product of the action of right representatives of $G_{L}$ in $G_{K}$, this does not depend on their choice.

Make two assumptions. First, assume that there is a surjective homomorphism of profinite groups

$$
\operatorname{deg}=\operatorname{deg}_{k}: G_{k} \rightarrow \widehat{\mathbb{Z}}
$$

Denote its kernel $G_{\tilde{k}}$. For an open subgroup $G_{K}$ of $G_{k}$, deg induces a surjective homomorphism

$$
\operatorname{deg}_{K}=\left|G_{k}: G_{K} G_{\tilde{k}}\right|^{-1} \operatorname{deg}_{k}: G_{K} \rightarrow \widehat{\mathbb{Z}} .
$$

Call any element of $G_{K}$ which is sent by $\operatorname{deg}_{K}$ to $1 \in \widehat{\mathbb{Z}}$ a frobenian with respect to $\operatorname{deg}_{K}$. Extensions of $K$ in $K \widetilde{k}$ will be called unramified. Second, assume that there is a homomorphism $v: A_{k} \rightarrow \widehat{\mathbb{Z}}$ whose image is $\mathbb{Z}$ or $\widehat{\mathbb{Z}}$ and such that the equality

$$
v\left(N_{L / K} A_{L}\right)=\left|G_{K} G_{\tilde{k}}: G_{L} G_{\tilde{k}}\right| v\left(A_{K}\right)
$$

holds for any open subgroup $G_{L}$ of any open subgroup $G_{K}$ of $G_{k}$.

For the pair (deg, $v$ ) we get a reciprocity map (not well defined, unless some further conditions are imposed) in the following way. For a finite extension $K$ of $k$ and a finite Galois extension $L / K$ and $\sigma$ in its Galois group, find any $\widetilde{\sigma} \in G(L \tilde{k} / K)$ such that $\operatorname{deg}(\widetilde{\sigma})$ is an integer $\geqslant 1$ (arithmetic orientation) and $\left.\tilde{\sigma}\right|_{L}=\sigma$. Denote by $\Sigma$ the fixed field of $\tilde{\sigma}$. Then $L \tilde{k}=\Sigma \tilde{k}$ and $L \tilde{k} / \Sigma$ is an unramified extension and $\tilde{\sigma}$ is a frobenian of $G_{\Sigma}$. Thus, every element $\sigma$ in the Galois group of $L / K$ can be lifted to a frobenian, after taking a composite with some appropriate finite extension $\Sigma / K$ depending on $\sigma$. Call $\pi_{K} \in A_{K}$ such that $v\left(N_{K / k} \pi_{K}\right)=\left|\widehat{\mathbb{Z}}: \operatorname{deg}\left(G_{K}\right)\right|$ a prime element of $A_{K}$. The element $\pi_{K}$ remains prime in all finite (unramified) subextensions of $K$ in $K \tilde{k}$.

Frobenians are mapped to prime elements with respect to the reciprocity map for unramified extensions, so then it is natural to define the (inverse to the) reciprocity map by the simple formula

$$
\Upsilon_{L / K}: \sigma \mapsto N_{\Sigma / K} \pi_{\Sigma} \bmod N_{L / K} A_{L},
$$

where $\pi_{\Sigma}$ is any prime element of $A_{\Sigma}$. This involves two indeterminacies with respect to the choice of $\widetilde{\sigma}$ and of $\pi_{\Sigma}$. 
Now we need two axioms for the $G$-module $A$ (axioms of CFT). The first is that the kernel of the norm map $N_{L / K}$ for cyclic extensions of prime degree is

$$
\left\{(1-\sigma) A_{L}: \sigma \in G(L / K)\right\} .
$$

The second axiom is a numerical (hence, a priori, not geometrical) property for finite abelian extensions $L / K$ : the index of $N_{L / K} A_{L}$ in $A_{K}$ equals the degree of $L / K$. Then one rather easily proves that $\Upsilon_{L / K}$ is a well defined homomorphism, and it induces an isomorphism

$$
G(L / K)^{\mathrm{ab}} \rightarrow A_{K} / N_{L / K} A_{L}
$$

satisfying all the standard functorial properties.

For a local field of mixed characteristic $k$ one can use the maximal unramified (in the usual sense) extension generated by roots of order prime to the residue characteristic as $\widetilde{k}$. One can also use other (ramified in the usual sense) $\widehat{\mathbb{Z}}$-extensions as $\tilde{k}$. Usually one uses the canonical (arithmetic) Frobenius automorphism to fix deg and takes $v$ as the usual surjective discrete valuation, then one gets the canonical local reciprocity map. We use the unique $\widehat{\mathbb{Z}}$-subextension of $\mathbb{Q}$ inside its maximal abelian extension $\mathbb{Q}^{\text {ab }}$ as $\widetilde{\mathbb{Q}}$. For a number field $k$ we use the composite of $k$ and $\widetilde{\mathbb{Q}}$ as $\tilde{k}$. The map $v$ is first defined on ideles as the composite of the product of the inverse to the local reciprocity homomorphisms (with the canonical local Frobenius automorphisms), which is then restricted to $G(\tilde{k} / k)$ and composed with deg. The reciprocity law for cyclotomic extensions of $\mathbb{Q}$ implies that $v$ factorises through idele classes. There is no canonical choice of a generator of $G(\widetilde{\mathbb{Q}} / \mathbb{Q})$, hence no canonical choice of deg, but the global reciprocity map does not depend on this choice, since using $a$ deg with $a \in \widehat{\mathbb{Z}}^{\times}$changes $v$ to $a v$ and the pairs (deg, $v$ ) and ( $a \mathrm{deg}, a v)$ define the same reciprocity map. In characteristic $p$ one uses the maximal constant field extensions; one can also use Artin-Schreier-Witt theory to explicitly get the $p$-primary part of CFT.

Explicit formulas for the (local) Hilbert pairing. Let $k$ contain $m$ roots of unity of order $m$, $\mu_{m}$. The Hilbert pairing

$$
(,)_{m}: k^{\times} / k^{\times m} \times k^{\times} / k^{\times m} \longrightarrow \mu_{m}
$$

is defined as $(a, b)_{m}=\sigma(c) / c$, where $c^{m}=b$ and $\sigma$ is the restriction on $k(\sqrt[m]{b}) / k$ of the image of $a$ with respect to the local reciprocity map. If one uses the Kummer map, then

$$
\begin{aligned}
(,)_{m}: k^{\times} / k^{\times m} \times k^{\times} / k^{\times m} & \longrightarrow H^{1}\left(G_{k}, \mu_{m}\right) \times H^{1}\left(G_{k}, \mu_{m}\right) \\
& \longrightarrow H^{2}\left(G_{k}, \mathbb{Z} / m \mathbb{Z}\right)={ }_{m} \operatorname{Br}(k) \simeq \mu_{m}
\end{aligned}
$$

after appropriate choices are made.

If $m$ is prime to the residue characteristic $p$, then the Hilbert pairing is a power of the tame symbol with its explicit linear algebra formula. When $m=p^{r}$, explicit formulas for the wild Hilbert symbol and its generalisations are far from linear algebra. Such formulas are at the heart of arithmetic. 
Two types of explicit formulas (for the wild Hilbert pairing and its generalisation to formal groups):

Partial/special explicit formulas: Artin-Hasse, Iwasawa, Coates-Wiles, Fontaine, PerrinRiou, Kato-Kurihara-Tsuji, Benois and others.

Full explicit formulas (for full values of the argument): Shafarevich, Vostokov, Brückner, Henniart, Fesenko, Kato. Because of its importance, let's include the Vostokov formula. Let $k$ be a finite extension of $\mathbb{Q}_{p}$ and let it contain a primitive $p^{n}$ th root $\zeta_{p^{n}}$ of unity, $p>2, n \geqslant 1$. Choose a prime element $\pi$ of $k$. Let $O_{0}$ be the ring of integers of the inertia subfield of $k, \operatorname{Tr}=\operatorname{Tr}_{O_{0} / \mathbb{Z}_{p}}$ and $\varphi$ be the Frobenius automorphism acting on $O_{0}$. Then for $\alpha, \beta \in k^{\times}$,

$$
(\alpha, \beta)_{p^{n}}=\zeta_{p^{n}}^{\operatorname{Tr} r e s} \Phi(A, B)(1 / S+1 / 2), \quad \Phi(A, B)=l(B) d A / A-l(A) \frac{1}{p} d B^{\Delta} / B^{\Delta},
$$

where $A, B \in O_{0}((X))^{\times}$are any series such that

$$
A(\pi)=\alpha, \quad B(\pi)=\beta,
$$

$S=S_{1}^{p^{n}}-1, S_{1} \in 1+X O_{0} \llbracket X \rrbracket$ is any series such that

$$
\begin{gathered}
S_{1}(\pi)=\zeta_{p^{n}} \\
l(A)=\log \left(A^{p} / A^{\Delta}\right) / p, \quad\left(\sum a_{i} X^{i}\right)^{\Delta}=\sum \varphi\left(a_{i}\right) X^{p i}
\end{gathered}
$$

see Chapter VII of [12]. Thus, this formula for the Hilbert pairing involves indeterminacies in relation to the choice of $\pi, A, B, S_{1}$. The right-hand side of the previous displayed formula is defined independently of CFT, it is called the Vostokov symbol and it can be used to establish part of CFT for Kummer extensions of $k$.

See [70] for a general review of explicit formulas, more information and references.

Remark. CFT is more than an abelian theory, it includes some information about abelian by finite extensions, the Shafarevich-Weil theorem: for an arithmetic field $k$ and finite $m / l / k, m / l$ abelian, $m / k, l / k$ Galois, the class of the group extension in $H^{2}(G(l / k)$, $\left.C_{l} / N_{m / l} C_{m}\right)$ of the exact sequence

$$
1 \longrightarrow C_{l} / N_{m / l} C_{m} \longrightarrow G(m / k) \longrightarrow G(l / k) \longrightarrow 1
$$

equals the image of the canonical class in $H^{2}\left(G(l / k), C_{l}\right)$. Koch-de Shalit's metabelian local class field theory, which is of SCFT type, may be viewed as one of the developments related to this theorem. A related development is general arithmetic non-abelian CFT, which is of GCFT type, it includes local theory for arithmetically profinite extensions, with its existence theorem and compatibility with ramification theory (Fesenko [7], IkedaSerbest [23-26]), and some global theory (Ikeda [22]).

Remark. For a discussion of some contrasting aspects of CFT and generalised Kummer theory see Section 4.2 of [55]. 


\section{From CFT to higher Kummer theory involving Milnor $K$-groups}

The Hilbert pairing satisfies the norm property: $(a, b)_{m}=1$ iff $b \in N_{k(\sqrt[m]{a}) / k} k(\sqrt[m]{a})^{\times}$. This implies the Steinberg property $(a, 1-a)_{m}=1$ for $a \neq 1$. So the Hilbert pairing is a symbol map and induces a map from Milnor $K_{2}(k)$ to $\mu_{m}$. If $m$ is the cardinality of roots of unity in $k$, the $m$ th Hilbert pairing is a universal continuous map from $K_{2}(k)$ to finite abelian groups, see e.g. Chapter IX of [12].

For any field $k$ of characteristic not dividing $m$, using the Kummer map and the cupproduct, one gets the 2-Kummer map:

$$
k^{\times} / k^{\times m} \times k^{\times} / k^{\times m} \longrightarrow H^{1}\left(G_{k}, \mu_{m}\right) \times H^{1}\left(G_{k}, \mu_{m}\right) \longrightarrow H^{2}\left(G_{k}, \mu_{m} \otimes \mu_{m}\right) .
$$

If $k$ is local, this is closely related to the Hilbert pairing. The 2-Kummer map satisfies the Steinberg property, hence induces the norm residue symbol

$$
K_{2}(k) / m K_{2}(k) \longrightarrow H^{2}\left(G_{k}, \mu_{m} \otimes \mu_{m}\right) .
$$

Key results about the norm residue symbol include: the norm residue symbol is an isomorphism for local and global fields (Tate, using CFT); the norm residue symbol is an isomorphism for all fields (Merkuriev-Suslin); the generalisation

$$
K_{r}(k) / m K_{r}(k) \longrightarrow H^{r}\left(G_{k}, \mu_{m}^{\otimes r}\right)
$$

of the norm residue symbol to Milnor $K_{r}$ is an isomorphism for all $r$ (Voevodsky, previous results of Suslin, Morel and Rost play an important role, for the first complete presentation of the proof see [17]).

Milnor $K$-groups of higher fields play a fundamental role in higher CFT.

A classical result of Matsumoto tells that for an infinite field $k$ its Milnor $K_{2}(k)$ is isomorphic to the fundamental group $\pi_{1}(G)$ for any split, simply connected, semi-simple, almost simple, algebraic group $G$ over $k$, not of symplectic type. Due to the work of Morel (see [57]) and Voevodsky, and a more recent work by Morel and Sawant, the sheaf of Milnor $K_{2}$ is canonically isomorphic to the motivic sheaf $\pi_{1}^{\mathbb{A}^{1}}(G)$ with $G$ the same as above.

\section{Three generalisations of CFT}

Each of the following three generalisations was started approximately half a century ago:

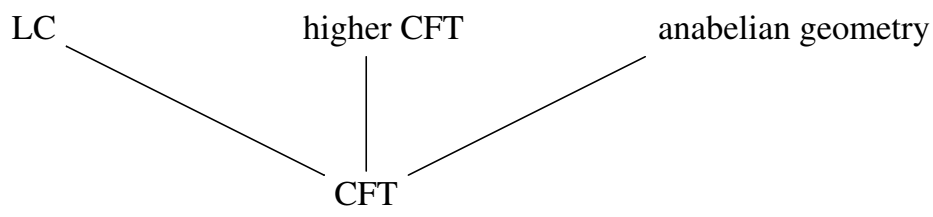


These generalisations use fundamental groups: the étale fundamental group in anabelian geometry, representations of the étale fundamental group (thus, forgetting something very essential about the full fundamental group) in Langlands correspondences and the (abelian) motivic $\mathbb{A}^{1}$ fundamental group (i.e. Milnor $K_{2}$ ) in two-dimensional (2d) higher class field theory.

\subsection{Langlands correspondences (LC)}

These theories are well known, they all use representation theory. Unlike CFT, whose modern expositions are not in need to involve any analytic results, the main arithmetic version of LC substantially involves analytic considerations. Its central analytic objects are two types of $L$-functions associated to Galois representations and to automorphic representations.

There are no known full analogues of Parts II, III of CFT in LC. For some very partial analogue of Part III of CFT in LC see e.g. Sections 1-2 of [1]. There is the higher ramification groups order formula for the Artin conductor, but it is rather not an LC analogue of Part IV of CFT.

Problem 1. Develop fuller LC analogues of Parts II-IV of CFT.

One way to characterise the correspondence in LC is to say that LC conjecturally classifies (irreducible) linear continuous representations of the Galois group (or related more complicated objects, such as the Weil or Weil-Deligne groups), using Artin $L$-functions and their generalisations, in terms of certain automorphic representations of local or adelic algebraic groups, using automorphic $L$-functions, in a way compatible with the classification of one-dimensional representations supplied by CFT. The $L$-functions, analytic objects, play fundamentally important role in the current form of LC for global fields and their completions. Recall that one can rewrite some of Part I of CFT for number fields as the property that for the $L$-function associated to a character of a finite abelian extension of a number field there is a unique primitive Hecke character of the number field with the same Hecke $L$-function. However, modern expositions of CFT do not use $L$-functions.

To some extent LC is a linear theory over abelian CFT. Since it is a representation theory, LC inevitably misses various important features of the full absolute Galois group that are not of linear representation type. Tannakian duality theoretically allows to recover a group from the tannakian category of its linear representations but this is difficult to do practically and, furthermore, not the full tannakian structure is taken care of by LC. For example, anabelian geometry uses the following two group theoretical properties of the absolute Galois group of a number field or of its nonarchimedean completion: each of its open subgroups is centre-free, each nontrivial normal closed subgroup $H$ of any open subgroup, with the property that $H$ is topologically finitely generated as a group, is open. ${ }^{5}$ How can one use these properties at the level of representations of these Galois groups?

\footnotetext{
${ }^{5}$ Profinite groups with the property that each nontrivial normal closed subgroup of any open subgroup is open are called hereditarily just-infinite, they form an important class in the classification of profinite groups, one representative is the Nottingham group and some of its hereditarily just-infinite subgroups can be realised as Galois groups of arithmetically profinite extensions of local fields, [6].
} 
L. Lafforgue wrote ${ }^{6}$ :

In the case of the absolute Galois group of a field, set-theoretic actions of this group correspond to separable extensions of the field. The world of its linear representations is so different that it is usually extremely difficult and deep to derive concrete consequences for separable extensions of results obtained for linear representations of this group

and

From the point of view of classifying toposes, going to linear representations is just a base change: instead of studying the classifying topos of a profinite group relatively to the absolute base, the topos of sets, you study it after base change with the classifying topos of the theory of vector spaces over some coefficient field. The fact that such a base change makes such a difference is extremely surprising.

The main conjectures of LC are stated over all global and local fields. There are important classes of representations of Galois groups naturally arising from torsion or division points of arithmetic objects or from appropriate cohomology groups. Over number fields and their completions, there has been progress in several directions, but we are still quite far from solutions of the key fundamental problems. Langlands wrote [46, p. 467]:

It appears that accidental, conditional phenomena are being used to establish general principles, a philosophically disagreeable circumstance.

Currently, the main arithmetic achievements in LC are of special type only. ${ }^{7}$ In the case of $\mathbb{Q}$, one of them is the work of Wiles and R. Taylor on modularity of $L$-functions of semistable elliptic curves over $\mathbb{Q}$ and its extensions to some small number fields. However, even the general Taniyama-Shimura conjecture over all number fields still stands unsolved, and its future general solution, as well as solutions of general conjectures of LC will be very different from the special methods used so far.

The $G L_{2}(\mathbb{Q})$ case is still open, 'We still don't know how to associate an Artin representation to a Galois Maass form' and 'the problem of Galois Maass forms is just the tip of an iceberg, ${ }^{8}$.

There are Harris-Taylor and Henniart's results about the local case for general linear groups using global methods, and still no fully local proof of local LC for all $G L_{n}$ is known.

Together with the correspondence issue (not an entirely analytic statement), the second most important issue in LC is functoriality (an analytic statement). Langlands wrote [46, p. 458]:

progress in functoriality, as a part of the theory of automorphic forms, has been largely analytic, exploiting very few arithmetic arguments [...] Relations between the analytic theory and the arithmetical have often been uneasy [...]

\footnotetext{
${ }^{6}$ From L. Lafforgue's email message, Feb 21, 2018.

${ }^{7}$ Compare with p. 467 of [46].

${ }^{8}$ From R. Taylor's email messages of Mar 4 and Mar 13, 2018.
} 
The proof of the fundamental lemma, a local statement, by Ngô, using Hitchin systems (which played an important role in geometric LC), was another highlight in all characteristics. Using it, Arthur established his trace formulas and various arithmetic applications.

The approach to functoriality through appropriate Fourier transforms and Poisson formulas was proposed for the first time in 2000 by Braverman and Kazhdan. L. Lafforgue, [41, 42], proposed a more precise formulation, both in characteristic zero and in positive characteristic, and proved the equivalence between functoriality and the existence of such non-additive Fourier transforms verifying a Poisson formula. In particular, these new Fourier transforms are defined and the associated Poisson formulas are proved in the function fields case. This reformulation asks for a definition of the Fourier transform on functional spaces for a general reductive algebraic group where one cannot use the obvious relation of $G L_{n}$ to matrices, without using the spectral decomposition results.

Fifty years after its start, most fundamental problems in arithmetic LC remain open. Is this a testament to the difficulty of the problems or to them not yet have been stated in the 'right' form? Work on this text revealed that researchers in LC often have a restricted knowledge of CFT, and in particular do not have a good knowledge of GCFT. Could that be one of key reasons why the most fundamental problems in arithmetic LC have not yet been solved? It is quite clear that some new fundamental arithmetic insights about LC are missing. Linear algebraic and geometric methods used in LC cannot fully cover or substitute various profound arithmetic issues, in particular those revealed by CFT and its other generalisations.

LC is non-abelian but not utmost non-abelian in comparison to anabelian geometry.

Question. Can the conjectures in arithmetic LC be fully established remaining solely inside the use of representation theory for adelic objects and Galois groups and class field theory? Or should one use more information about the absolute Galois group of global and local fields, which cannot be reached via representation theory, e.g. such as in anabelian geometry?

The third important prediction of LC is that the $L$-factors of zeta functions of regular proper arithmetic schemes coincide with appropriate automorphic $L$-functions, [46]. The best result in this direction is the theorem of Wiles and its extension for all elliptic curves of $\mathbb{Q}$. See Section 6 below for a $2 \mathrm{dAAG}$ approach to the zeta functions.

In positive characteristic there has been much more progress using geometrical tools which are not available in the standard approaches to LC over number fields. The local $G L_{r}$ case was established by Laumon, Stuhler and Rapoport for $r \geqslant 2$. The global $G L_{r}$ case was established by Drinfeld for $r=2$, and by L. Lafforgue for all $r$ (see [40]) using Drinfeld modules and $F H$-sheaves ${ }^{9}$. Further important work on functoriality by V. Lafforgue, using in particular ideas from the geometric theory, described how to go from

\footnotetext{
${ }^{9}$ Named so by G. Harder and D. Kazhdan in [18], F for Frobenius, H for Hecke, instead of the original name shtuki (pl.). One of the main translations of shtuka (sing.) is whatsit, thingummy, gizmo.
} 
the automorphic side to the Galois side in positive characteristic for arbitrary reductive algebraic groups, [43]. To establish functoriality one currently has to go through Galois representations.

L. Lafforgue wrote ${ }^{10}$ :

the methods used to construct LC for function fields or pieces of this correspondence (for number fields) are highly non-explicit, as they consist in considering and studying $l$-adic cohomology spaces of sophisticated algebraic varieties. Galois representations constructed as components of cohomology spaces are not at all objects one can describe.

Remark. Inaba's work on matrix Artin-Schreier theory [27,28] describes all finite Galois extensions of degree a power of $p$ of fields of characteristic $p$. It may lead to a simplified $p$-primary part of LC in characteristic $p$.

The geometric Langlands correspondence ${ }^{11}$, further away from arithmetical aspects of CFT, was initiated by Deligne, Drinfeld and Laumon and developed by BeilinsonDrinfeld, Gaitsgory, Frenkel, and others. Hitchin systems proved to be very useful for this theory. There are various links between geometric Langlands correspondence and physics such as the paper of Kapustin-Witten. A categorical geometric Langlands correspondence was proposed by Gaitsgory in [16]. While one of the key features of CFT is its functoriality with respect to finite separable base change, both LC and geometric LC currently experience difficulties with its analogues. Basic problems remain unsolved or even non-stated, e.g. functoriality; partial progress uses the theta sheaf constructed by Lysenko, [47-49]. It is still not clear, even conjecturally, what happens when one passes from a curve to its finite étale cover, and the ramified case remains substantially open too. Frenkel [15] discusses possible analytic approached to automorphic fundings on complex algebraic curves; see also [3].

Remark. Apparently, no significant development within LC is parallel to (or can be viewed as a generalisation of or parallel to) GCFT. Thus, in the arithmetic Langlands correspondence, 50 years after its start, we are still in the 'pre-Takagi' stage, similar to where we were 100 years ago in CFT.

Problem 2. Find a version of enhanced arithmetic LC parallel to GCFT.

Problem 3. Find a version of enhanced LC parallel to SCFT but which works over all number fields.

Problem 4. Find a version of enhanced LC parallel to some of post-cohomological CFT, thus circumventing the problem of using non-abelian cohomology.

\footnotetext{
${ }^{10}$ From L. Lafforgue's email message, Feb 21, 2018.

${ }^{11}$ For the most recent review see [14].
} 
Note that since LC is a linear theory whereas CFT is not, the asked enhancements of LC have to be non-linear. Hopefully, better knowledge and use of GCFT can be helpful for further progress in LC. Langlands emphasised the importance of Hasse's Klassenkörperbericht. $^{12}$

Concerning Problem 3, such a mixture is a property of Mochizuki's IUT theory, a further development of anabelian geometry. This theory is of special type and works over all number fields.

A very different approach to the description of certain non-commutative Galois extensions of function fields of curves over finite fields was started by Ihara, [21]. For example, this theory describes the maximal unramified cover of the projective line over $\mathbb{F}_{p^{2}}$ minus three points which is at most tamely ramified at the three points and in which some special finite set of points decomposes completely, in terms of subgroups of finite index of the quotient of the subgroup of $S L_{2}(\mathbb{Z}[1 / p])$ congruent to the identity mod 2 by its cyclic subgroup of order 2 .

\subsection{2d (and higher) CFTs}

Classical Lang's geometric CFT for function fields of varieties over finite fields used commutative algebraic groups over finite fields and their isogenies, [44, 45]. Even though one can deduce some of its main results from higher CFT, see [34], the point of view of geometric CFT on obtaining abelian étale coverings as pullbacks from isogenies of commutative group schemes has a larger geometric content.

CFT for $n$-dimensional schemes take into account CFT at the $(n-1)$-residue level. Almost all higher CFTs typically use Milnor $K$-groups. They are of GCFT type. A large number of main properties in higher CFT has been established.

Cohomological higher CFT was developed by Kato (higher local fields), [32, 33], while local-global and global theories were developed by Kato, Sh. Saito, Kato-Sh. Saito, $[34,35]$, Bloch, Spieß, Jannsen-Sh. Saito. They generalise some of the cohomological approaches to CFT. The local part of Kato's theory works with $H^{3}\left(G_{F}, \mu_{m} \otimes \mu_{m}\right)$ instead of the $m$-torsion part of the Brauer group. The cohomological 2dCFT is an $H^{3}$-theory. Other $1 \mathrm{~d}$ cohomological approaches cannot be (easily) generalised to $2 \mathrm{~d}$. In particular, for a finite Galois extension $L / F$ the homomorphism $K_{2}(F) \longrightarrow K_{2}(L)^{G(L / F)}$ is neither injective nor surjective in general, so the $1 \mathrm{~d}$ class formations approach does not generalise directly.

An explicit higher local CFT in any characteristic, including the most difficult case of mixed characteristic fields, was constructed in [4], it generalised the Neukirch method and worked with several topologies on Milnor $K$-groups of higher fields and their quotient $K^{t}$, making use of a higher local fields version of the Vostokov symbol, [70], and of the ArtinSchreier-Witt pairing. These two pairings are higher analogues of explicit formulas of Part III of CFT and exist independently of CFT.

\footnotetext{
${ }^{12}$ In two email messages of Feb 9, 2016 and Feb 24, 2018.
} 
Remark. For the $p$-primary part of CFT in characteristic $p$ one cannot use Kummer theory. However, the Kawada-Satake method and its generalisations [13], using Witt theory, makes the $p$-primary part the easiest part of CFT in characteristic $p$. The nature of existence theorem in positive characteristic is clarified in [13]: it corresponds to topological reflexivity of (generally non-locally compact) groups with respect to a generalised explicit pairing. An explicit approach to higher CFT in positive characteristic by Parshin, [62, 63], is a higher local fields extension of the Kawada-Satake method, [36], several errors and gaps in [62,63] were fixed in [13].

Perhaps, the shortest way to state the reciprocity map of 2dCFT is probably this: the 2 d reciprocity map for a $2 \mathrm{~d}$ global field $K$ is a continuous homomorphism

$$
\Psi_{K}: K_{2}^{t}(\mathbf{A}) /\left(K_{2}^{t}(\mathbf{B})+K_{2}^{t}(\mathbf{C})\right) \longrightarrow G_{K}^{\mathrm{ab}},
$$

where $\mathbf{A}$ is $2 \mathrm{~d}$ geometric adeles associated to a proper regular model of $K, \mathbf{B}$ is its subspace corresponding to divisors and $\mathbf{C}$ corresponds to closed points,

$$
K_{2}^{t}(\mathbf{A})=K_{2}(\mathbf{A}) /\left(\cap_{l \geqslant 1} l K_{2}(\mathbf{A})\right)
$$

and $K_{2}^{t}(B), K_{2}^{t}(C)$ are images in $K_{2}^{t}(A)$ of $K_{2}(B)$ and $K_{2}(C)$, [8, Part II].

All these 2dCFT are GCFT, they do have analogues of Parts II, III of CFT, but not of Part IV due to lack of a satisfactory $2 \mathrm{~d}$ ramification theory satisfying analogues of each of (a), (b), (c) at the end of Section 3. There are many different approaches to $2 \mathrm{~d}$ local ramification theory, including those by Kato, Hyodo, Zhukov, Fesenko, Kato-T. Saito, Borger, Abbes-T. Saito, each having merits and disadvantages, and none capable to serve as a comprehensive $2 \mathrm{~d}$ ramification theory.

Problem 5. Develop a general ramification theory for surfaces compatible with $2 d C F T$ and taking into careful account ramification theory at the one-dimensional residue level.

An approach to parts of higher global CFT in any characteristic, which uses the étale fundamental group and does not use Milnor $K$-groups, was developed by Wiesend, $[73,74]$. It analyses which finite compatible covers of closed points and curves on a regular arithmetic scheme correspond to a finite Galois cover of the scheme. This leads to a simplified approach to parts of global higher CFT. Unusually for CFT, this approach does not (yet) have local and local-to-global parts. ${ }^{13}$

Remark. Unlike the classical one-dimensional case, 2dCFT is somehow separated from geometry. The distance between full $2 \mathrm{~d}$ geometric adeles $\mathbf{A}$ and the function field $K$ is two-step, intermediate local-global adelic objects are $\mathbf{B}$ and $\mathbf{C}$. The Picard group of a proper regular model of $K$ is isomorphic to $\mathbf{B}^{\times} /\left(K^{\times} \cdot\right.$ units $)$.

\footnotetext{
${ }^{13}$ Some corrections, extensions and applications of Wiesend's approach were later produced by Schmidt and Kerz, $[37,38]$.
} 
Remark. No significant development within higher CFT is parallel to (or can be viewed as a generalisation of) SCFT.

Problem 6. Develop a special higher CFT which uses torsion structures, to provide new insights into $2 d C F T$.

We observe some analogy between the role of special and general approaches to CFT and to its generalisations such as LC and higher CFT. In particular, the main achievements of LC in the number field case are of special type.

\subsection{Anabelian geometry}

The contributors include Neukirch, Iwasawa, Ikeda, Uchida (for 1d fields, it uses the computation of the Brauer group of arithmetic fields); Pop (birational anabelian geometry for finitely generated fields); Belyi; Nakamura, Tamagawa, Mochizuki (hyperbolic curves over finite fields and subfields of local number fields); Stix (positive characteristic).

A very different birational geometry for function fields of varieties of dimension $>2$ over $\mathbb{C}$, which uses Milnor $K$-groups, was pioneered by Bogomolov and developed by Bogomolov-Tschinkel, and contributed to later by Pop and Topaz.

There is no analogue of Parts II, III of CFT in anabelian geometry, but there might be some analogue of Part IV of CFT.

Anabelian geometry is a sort of utmost non-abelian and non-linear theory, working with full topological groups such as the absolute Galois groups and fundamental groups of hyperbolic curves (smooth projective geometrically connected curve whose Euler characteristic is negative). Rigidity of certain Galois and fundamental groups is a key feature of anabelian geometry.

Tamagawa's theorem states that for two non-proper hyperbolic curves $C_{1}, C_{2}$ over a finitely generated field $k$ over $\mathbb{Q}$, the natural morphism from $k$-isomorphisms of $k$ schemes $C_{1}$ to $C_{2}$ to continuous $G_{k}$-isomorphisms of their étale fundamental groups modulo inner automorphisms of the étale fundamental group of $C_{2} \times_{k} k^{\text {alg }}$ is bijective, [67]. One of Mochizuki's theorem extends this property to all hyperbolic curves, [51]. Another stronger theorem of Mochizuki states that the natural morphism from dominant $k$-morphisms of hyperbolic curves $C_{1}, C_{2}$ over a subfield $k$ of a field finitely generated over $p$-adic numbers to open continuous $G_{k}$-homomorphisms of their étale fundamental groups, considered up to composition with an inner automorphism of the étale fundamental group of $C_{2} \times_{k} k^{\text {alg }}$, is bijective, [52,53].

Mono-anabelian geometry developed by Mochizuki further extends anabelian geometry. It includes strong results on algorithmical reconstruction of an arithmetic object from certain fundamental groups. One of the main theorems of mono-anabelian geometry is Mochizuki's algorithmic reconstruction of $k$ and the function field of a hyperbolic curve of strictly Belyi type over $k$, with $k$ either a number field or its nonarchimedean completion, from the étale fundamental group of the curve, [54, Part III]. Recall that in general one cannot reconstruct a finite extension of $\mathbb{Q}_{p}$ from its absolute Galois group, thus the 
previous theorem demonstrates that using the next dimension by involving certain hyperbolic curves over the field fundamentally improves the situation.

A fundamental related development is the study of the (profinite) Grothendieck-Teichmüller group (GT) in relation to its subgroup $G_{\mathbb{Q}}$. The previous role of Grothendieck's theory of dessins d'enfants and the contributions of Drinfeld, Lochak and Schneps and others are well known. A recent paper of Mochizuki-Hoshi-Minamide [20] shows that GT is, up to $S_{n+3}$, just the group of outer automorphisms of the étale fundamental group of the $n$-dimensional configuration space, $n>1$, associated to a hyperbolic curve of genus 0 with 3 punctures over an algebraically closed field of characteristic zero. This result suggests $G_{\mathbb{Q}}$ as a proper subgroup of GT. One of corollaries, by Minamide and Nakamura, is the description of the profinite Grothendieck-Teichmüller group as the outer automorphism group of the mapping class group of a topological torus with two marked points, [50]. Another recent result by Tsujimura establishes a surjective homomorphism from the (largest) $p$-adic Grothendieck-Teichmüller group to $G_{\mathbb{Q}_{p}}$ whose restriction on $G_{\mathbb{Q}_{p}}$ is the identity map, [69].

Remark. The anabelian geometry viewpoint provides a new understanding of the role of the class formations in axiomatic approaches to CFT. Checking the validity of axioms of CFT requires the use of the ring structure, while the CFT mechanisms, i.e. deducing the reciprocity map and other properties from the axioms of CFT, is purely group theoretical. It is interesting to note that Neukirch's explicit approach to CFT (Section 3), was partially motivated by his experience in anabelian geometry for number fields.

\section{Three generalisations of CFT in arithmetic of elliptic curves}

In this last section we look at the particular example of an elliptic curve $E$ over a number field $k$. Choose a proper regular model $\&$ flat over the ring of integers of $k$. Denote $K=$ $k(E)=k(\mathcal{E})$.

LC: here the main achievement is of Wiles-Taylor et al. for elliptic curves over $\mathbb{Q}$. It is of special type. The associated Galois and $l$-adic representations of $G_{k}$ use torsion points of $E$ over a separable closure of $k$. Current approaches to LC for elliptic curves over number fields work with the generic fibre of $\mathcal{E}$ or with special fibres of $\mathcal{E}$, but rather not with the full arithmetic surface $\mathcal{E}$ as a two-dimensional geometric object.

2dCFT: The $2 \mathrm{~d}$ reciprocity map

$$
\Psi_{K}: K_{2}^{t}(\mathbf{A}) /\left(K_{2}^{t}(\mathbf{B})+K_{2}^{t}(\mathbf{C})\right) \longrightarrow G_{K}^{\mathrm{ab}}
$$

is the outcome of two global reciprocity laws with respect to $K_{2}^{t}(\mathbf{B})$ and $K_{2}^{t}(\mathbf{C})$, associated to curves and to points of $\mathcal{E}$, of $2 \mathrm{~d}$ adelic product of $2 \mathrm{~d}$ local reciprocity maps

$$
\Psi_{F}: K_{2}^{t}(F) \longrightarrow G_{F}^{\mathrm{ab}},
$$

where $F$ is a 2 d local field associated to a point on a curve on $\mathcal{E}$. The local reciprocity map sends $K_{2}^{t}$-prime elements such as $\left\{t_{1}, t_{2}\right\}$, where $t_{2}$ is a local parameter of $F$ and $t_{1}$ is a lift 
to $F$ of a local parameter of its residue field, to an automorphism of the maximal abelian extension of $F$ which when restricted to the maximal prime-to- $p$-cyclotomic extension of $F$ is equal to its Frobenius automorphism over $F$. A generalisation of the 1d Neukirch method works for $K_{2}^{t}$-objects, [4].

Anabelian geometry: one can work with various hyperbolic curves associated to $E$, e.g. $X=E \backslash\{0\}$ over $k$, and with the homomorphism $\pi_{1}(X) \rightarrow G_{k}$. From the homomorphism $\pi_{1}(X) \rightarrow G_{k}$ one can algorithmically recover $k(X)$ and the fields $k$ and completions $k_{v}$ of $k$, [54]. It is well known that from $G_{k_{v}}$ one cannot in general recover $k_{v}$.

Anabelian geometry is intensively used in Mochizuki's $I U T=$ arithmetic deformation theory and its applications to some of the abc inequalities, and the Szpiro and Vojta conjectures, $[55,56]$. It is interesting to observe that similarly to the Neukirch explicit CFT and the Vostokov symbol in explicit formulas for the Hilbert pairing, IUT involves several indeterminacies at its crucial stage of multi-radial representation. IUT uses generalised Kummer theory and the computation of the local Brauer group, it does not use anything else from CFT. It works with values of certain nonarchimedean functions (étale theta functions) at torsion points, in this respect it is nearer to SCFT; on the other hand, it works over any number field and in this respect it is nearer to GCFT. ${ }^{14}$

Informally speaking, IUT deals with Galois groups as tangent bundles, see the beginning of Sections 2.6 and 4.3 (ii) of [55]. To a certain degree, global class field theory does kind of the same with abelian Galois groups: abelian Galois groups over a global field correspond to idele classes, while adeles are dual to generalised differential forms.

$2 d A A G=2 d$ adelic analysis and geometry studies properties of the zeta function of $\mathcal{E}$ by involving $2 \mathrm{~d}$ analytic adeles and $2 \mathrm{~d}$ zeta integrals. ${ }^{15}$ Recall that the (Hasse) zeta function of $\varepsilon$ is

$$
\zeta_{\mathcal{E}}(s)=\prod_{x}\left(1-|k(x)|^{-s}\right)^{-1}
$$

$x$ runs through all closed points of $\mathcal{E}$. Its conjectural functional equation is

$$
c_{\mathcal{E}}^{2-s} \zeta_{\mathcal{E}}(2-s)^{2}=c_{\mathcal{E}}^{s} \zeta_{\mathcal{E}}(s)^{2}
$$

with some positive rational $c_{\mathcal{E}}$ not depending on the archimedean data; note the absence of the $\Gamma$-factors. This functional equation is known to hold if $k=\mathbb{Q}$ by the work of Wiles and Taylor and others. Dependent on the generic fibre $E$ of $\&$ only, the (Hasse-Weil) zeta function $\zeta_{E}(s)$ of $E$ satisfies the equation $\zeta_{\mathcal{E}}(s)=n_{\mathcal{E}}(s) \zeta_{E}(s)$ where the factor $n_{\mathcal{E}}(s)$ is the product of finitely many zeta functions of affine lines over finite fields, corresponding to what happens at singular fibres of $\mathcal{E}$. If $\mathcal{E}$ is the global minimal Weierstraß model of $E$, then $n_{\varepsilon}(s)=1$. The $L$-factor (i.e. the Hasse-Weil $L$-function of $E$ ) is the denominator of $\zeta_{E}$ :

$$
\zeta_{E}(s)=\zeta_{\mathbb{P}^{1}(k)}(s) / L_{E}(s)
$$

\footnotetext{
${ }^{14}$ See also Remark 2.3.3 of [56, Part IV] and Section 4.2 of [55].

${ }^{15}$ See Sections I, J, K of https://www.maths.nottingham.ac.uk/personal/ibf/mp.html.
} 
Thus, the conjectural functional equation of $L_{E}$ is more complicated than that of $\zeta_{\mathcal{E}}$, and its Gamma-factor has its origin due to the 1d Gamma-factor in the functional equation of $\zeta_{k}(s) \zeta_{k}(s-1)$.

The $L$-factors of zeta-functions of proper regular arithmetic schemes are like micro/ quantum objects, whose non-commutative study requires the use of representation theory, once developed for the needs of quantum mechanics and then finding applications in various parts of mathematics including number theory. Thus, in some sense they are more complicated objects than the zeta functions of proper regular arithmetic schemes which are like macro objects and can be studied without using representation theory, using commutative (but higher dimensional) methods.

Now we consider the role of zeta integrals in the classical setting of the Iwasawa-Tate theory, and in $2 \mathrm{dAAG}$ and LC.

Zeta integrals. Classically, i.e. in the Iwasawa-Tate theory, [29-31, 68], a completed zeta function is written as a zeta integral

$$
\int_{\mathbb{A}_{k}^{\times}} f(\alpha)|\alpha|^{s} d \mu_{\mathbb{A}_{k}^{\times}}(\alpha)
$$

over ideles $\mathbb{A}_{k}^{\times}$of the product of a Bruhat-Schwartz function $f$ and the module function raised to complex power $s$, against a nontrivial translation invariant measure on the locally compact group of ideles. More generally, one can replace $|\alpha|^{s}$ with an arbitrary quasicharacter $\chi$ of $\mathbb{A}_{k}^{\times}$vanishing on $k^{\times}$. To deduce the functional equation and meromorphic continuation of the zeta integral one uses dualities of additive adeles and objects from appropriate $1 \mathrm{~d}$ CFT, such as $\mathbb{A}_{k}^{\times}, \mathbb{A}_{k}^{\times} / k^{\times}$, but not $1 \mathrm{~d}$ CFT itself.

Recall that some of Part I of CFT for global fields is equivalent to the following property: the $L$-function of a global field associated to a character of its absolute Galois group, after being appropriately completed with Gamma-factors is equal to

$$
\int_{\mathbb{A}_{k}^{\times}} f(\alpha) \chi(\alpha) d \mu_{\mathbb{A}^{\times}}(\alpha)
$$

for an appropriate quasi-character $\chi$ of $\mathbb{A}_{k}^{\times}$vanishing on $k^{\times}$. For one-dimensional objects of number theory, the three zeta/ $L$-functions coincide: scheme theoretic, abelian Galois representation theoretic and abelian automorphic representation theoretic.

The role of appropriately generalised zeta integrals in $2 \mathrm{dAAG}$ and $\mathrm{LC}$ is fundamental. $2 \mathrm{dAAG}$ includes a $2 \mathrm{~d}$ zeta integral presentation

$$
\int_{\mathbb{A}^{\times} \times \mathbb{A}^{\times}} f(\alpha)\|\alpha\|^{s} d \mu_{\mathbb{A}^{\times} \times \mathbb{A}^{\times}}(\alpha)
$$

of the square of $\zeta_{\mathcal{E}}(s)$ times appropriately scaled squares of 1d zeta integrals of finitely many horizontal curves on $\mathcal{E}$, where $f$ is a 2 d Bruhat-Schwartz function and \|\|$^{s}$ is a twisted module homomorphism, against a $2 \mathrm{~d}$ translation invariant measure on the multiplicative group of $2 \mathrm{~d}$ analytic adeles $\mathbb{A}$ (which are fundamentally different from the 
$2 \mathrm{~d}$ geometric adeles $\mathbf{A}$ of $\mathcal{E}$ and from the $1 \mathrm{~d}$ adeles $\mathbb{A}_{k}$ ), [8, Part II]. This study has applications to the meromorphic continuation and functional equality of $\zeta_{\mathcal{E}}(s)$ via a $2 \mathrm{~d}$ theta-formula and resulting boundary term and mean-periodicity conjecture, $[8,66]$; to the GRH for $\zeta_{\varepsilon}(s)$ via the Laplace-Carleman spectrum of the boundary term, [8, 65]; and to the BSD conjecture via the boundary term and an interaction between the multiplicative groups of $2 \mathrm{~d}$ geometric and analytic adeles, [8, Part III]. In particular, an interaction between the multiplicative groups of the two adelic structures

$$
K_{1}(\mathbf{A}) \times K_{1}(\mathbb{A}) \rightarrow K_{2}(\mathbf{A}),
$$

originating from explicit existence theorem in 2dCFT, an analogue of Part II of CFT, leads to a relation between the analytic and geometric adelic structures that can be viewed as an adelic lift of the well known conjecture about the two ranks of $E$, [8, Part III]. This seems to be the only general (i.e. working over all global fields) approach to the study of special values of the zeta function $\zeta_{\mathcal{E}}(s)$. All this information about $\zeta_{\mathcal{E}}(s)$ implies the corresponding information about $L_{E}(s)$. Thus, similar to the property of mono-anabelian geometry mentioned in Section 5.3 , the use of $2 \mathrm{~d}$ objects in $2 \mathrm{dAAG}$ allows one to see deeper properties of the zeta-function and $L$-function of an elliptic curve over a global field. Without using the hard part of the theory of [8, Part II] the mean-periodic conjecture was extended by Fesenko, Ricotta and Suzuki to the zeta functions of all proper regular arithmetic schemes in [11]. It directly tells, without looking at automorphicity of the $L$-factors, what is an equivalent condition for the zeta function to have a meromorphic extension and functional equation.

For the $L$-function of an irreducible $G L_{n}$-representation of the absolute Galois group $G_{k}$ of a global field $k$, its conjectural automorphicity, due to the converse theorems, is closely related to the following conjectural property: its completed $L$-function and its twists by appropriate characters, after multiplying with appropriate Gamma-factors, is equal to an appropriate zeta integral, for an appropriate $M\left(n, \mathbb{A}_{k}\right)$-Bruhat-Schwartz function $f$,

$$
\int_{G L_{n}\left(\mathbb{A}_{k}\right)} f(\alpha) c(\alpha)|\operatorname{det}(\alpha)|^{s} d \mu_{G L_{n}\left(\mathbb{A}_{k}\right)}(\alpha) .
$$

The additional factor

$$
c(\alpha)=\int_{G L_{n}\left(\mathbb{A}_{k}\right)^{1} / G L_{n}(k)} g_{1}(\gamma \alpha) g_{2}(\gamma) d \mu(\gamma) \text { for } n>1
$$

involves two cuspidal functions $g_{i}$. Here, $G L_{n}\left(\mathbb{A}_{k}\right)^{1}$ is the preimage of the unit circle with respect to the determinant, and a complex valued cuspidal function $g$ is a smooth (in the adelic sense) function such that the $\alpha$-variable integral of $g(\alpha \beta)$ over $U_{r, n}\left(\mathbb{A}_{k}\right) / U_{r, n}(k)$ is zero for all $\beta \in G L_{n}\left(\mathbb{A}_{k}\right)$, where $U_{r, n}$ is the group of block upper-triangle matrices with two diagonal blocks being the identity matrices of order $r$ and $n-r, 1 \leqslant r \leqslant n-1$. For $n \geqslant 4$ one may need a similar property of zeta integral presentation to hold for the twists of the $L$-function by appropriate $L$-functions of irreducible $G L_{m}$-representations for all $2 \leqslant m \leqslant n-2$, to use the converse theorems. 
It is quite possible that such complex functions should include the Kurokawa-Selberg zeta function of the fundamental group of hyperbolic curves. This zeta function is defined in Section 1 of [39] which proposes a conjectural relation of three types of zeta/ $L$-functions with the Kurokawa-Selberg zeta function.

Here are some relations between the three generalisations of CFT and their further developments:

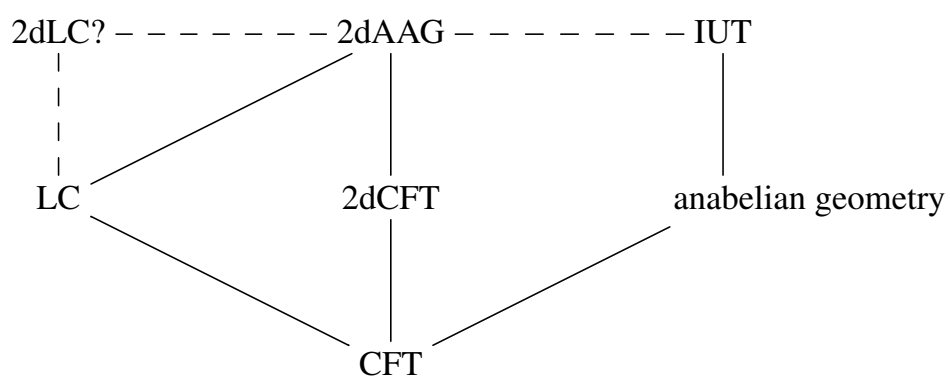

$2 \mathrm{dLC}$ should exist but not much is known about it. An object, generalising the quotient $G L_{2}(\mathbb{A}) / G L_{2}(k)$ in $1 \mathrm{~d}$ case, certain functions on which may serve as $2 \mathrm{~d}$ automorphic functions of $\varepsilon$ is described in the last section of [9]. It is expected that local 2dLC will use higher translation invariant measure and integration on algebraic groups over $2 \mathrm{~d}$ local fields in $[9,58,59,71]$.

Relations between 2dAAG and IUT include, in addition to those mentioned in [10], the following analogy. IUT uses (after fixing a prime number $l$ ) two fundamental symmetries: additive geometric and multiplicative arithmetic. 2dAAG uses adelic structures on arithmetic surfaces: geometric adeles (their additive structure is related to Zariski cohomology and the intersection pairing, their $K_{2}$-structure is important for 2dCFT) and multiplicative analytic adeles $\mathbb{A}^{\times}$(used to study the $2 \mathrm{~d}$ zeta integral, zero cycles).

As for potential relations between IUT and LC, one of the key activities in anabelian geometry is the restoration of ring structure, i.e. the second operation of addition, when the multiplicative structure is already known. It is interesting to compare with [41] which reformulates the functoriality in LC as a problem to find for an arbitrary reductive algebraic group an analogue of the relation between the group $G L_{n}$ and the ring of square matrices of order $n$.

Problem 7. Find more direct relations between the generalisations of CFT. Use them to produce a single unified generalisation of $C F T .^{16}$

Such relations, when found, may lead to new approaches to LC which are parallel to general CFT, or, at least have some mixture of features of special CFT but work over all number fields, as they do in IUT. They may even lead to a unified powerful generalisation of CFT which specialises to its three generalisations.

\footnotetext{
${ }^{16}$ Stated as the result of e-communication with V. Shokurov.
} 
Acknowledgments. I am grateful to L. Lafforgue and Sh. Mochizuki for many valuable discussions, remarks and help, and to D. Arinkin, A. Beilinson, V. Drinfeld, E. Frenkel, V. Lafforgue, R. Langlands, S. Lysenko, V. Shokurov for their comments, suggestions and answers. I thank the referees for their useful comments, corrections and inspiring reaction to this text.

The first, short version of this text was written on the occasion of delivering a talk at a meeting of Symmetries and Correspondences in Oxford in February 2018. Further talks related to this text were given in July-August 2018 in Kyoto and at the December 2018 conference on intra-disciplinary developments in geometry.

Funding. This work was partially supported by EPSRC Programme grant EP/M024830.

\section{References}

[1] J. Arthur, Automorphic representations and number theory. In 1980 Seminar on Harmonic Analysis, McGill University (Montréal, 1980), pp. 3-54, Canad. Math. Soc. Conf. Proceedings 1. Amer. Math. Soc., Providence, RI, 1981. Available from http://www.math.toronto.edu/ arthur/pdf/11.pdf

[2] E. Artin and J. Tate, Class field theory. W. A. Benjamin, Inc., New York-Amsterdam, 1968 Zbl 0176.33504 MR 0223335

[3] P. Etingof, E. Frenkel, and D. Kazhdan, An analytic version of the Langlands correspondence for complex curves, 2020 arXiv:1908.09677v3

[4] I. Fesenko, Class field theory of multidimensional local fields of characteristic 0 with residue field of positive characteristic. Part I. Algebra i Analiz 3 (1991), no. 3, 165-196; English transl. in St. Petersburg Math. 3 (1992), no. 3, 649-678 Zbl 0791.11063 MR 1150559;

Part II. Algebra i Analiz 3 (1991), no. 5, 168-189; English transl. in St. Petersburg Math. J. 3 (1992), no. 5, 1103-1126 Zbl 0770.11052 MR 1186241

[5] I. Fesenko, Local class field theory: the perfect residue field case. Izv. Ross. Akad. Nauk Ser. Mat. 57 (1993), no. 4, 72-91; English transl. in Russ. Acad. Scienc. Izvest. Math. 43 (1994), no. 1, 65-81 Zbl 0826.11056 MR 1243352

[6] I. Fesenko, On just infinite pro- $p$-groups and arithmetically profinite extensions of local fields. J. Reine Angew. Math. 517 (1999), 61-80 Zbl 0997.11107 MR 1728547

[7] I. Fesenko, Nonabelian local reciprocity maps. In Class field theory-its centenary and prospect (Tokyo, 1998), pp. 63-78, Adv. Stud. Pure Math. 30, Math. Soc. Japan, Tokyo, 2001 Zbl 1039.11085 MR 1846451

[8] I. Fesenko, Analysis on arithmetic schemes. Part I. Docum. Math. Kazuya Kato's fiftieth birthday (2003), Extra. Vol., 261-284 Zbl 1130.11335 MR 2046602; Part II. J. K-Theory 5 (2010), no. 3, 437-557 Zbl 1225.14019 MR 2658047; Part III. Available from https://www. maths.nottingham.ac.uk/personal/ibf/a3.pdf

[9] I. Fesenko, Adelic approach to the zeta function of arithmetic schemes in dimension two. Mosc. Math. J. 8 (2008), no. 2, 273-317 Zbl 1158.14023 MR 2462437

[10] I. Fesenko, Arithmetic deformation theory via arithmetic fundamental groups and nonarchimedean theta-functions, notes on the work of Shinichi Mochizuki. Eur. J. Math. 1 (2015), no. 3, 405-440 Zbl 1416.11119 MR 3401899 
[11] I. Fesenko, G. Ricotta, and M. Suzuki, Mean-periodicity and zeta functions. Ann. Inst. Fourier (Grenoble) 62 (2012), no. 5, 1819-1887 Zbl 1305.11079 MR 3025155

[12] I. Fesenko and S. V. Vostokov, Local fields and their extensions. With a foreword by I. R. Shafarevich. Second edn., Transl. Math. Monogr. 121. Amer. Math. Soc., Providence, RI, 2002 Zbl 1156.11046 MR 1915966

[13] I. Fesenko, S. V. Vostokov, and S. H. Yoon, Generalised Kawada-Satake method for Mackey functors in class field theory. Eur. J. Math. 4 (2018), no. 3, 953-987 Zbl 1426.19004 MR 3851125

[14] E. Frenkel, Langlands program, trace formulas, and their geometrization. Bull. Amer. Math. Soc. (N.S.) 50 (2013), no. 1, 1-55 Zbl 1329.11121 MR 2994994

[15] E. Frenkel, Is there an analytic theory of automorphic functions for complex algebraic curves? SIGMA Symmetry Integrability Geom. Methods Appl. 16 (2020), Paper No. 042, 31pp Zbl 1446.14005 MR 4098670

[16] D. Gaitsgory, Outline of the proof of the geometric Langlands conjecture for $G L_{2}$. Astérisque (2015), no. 370,1-112 Zbl 1406.14008 MR 3364744

[17] C. Haesemeyer and C. A. Weibel, The norm residue theorem in motivic cohomology. Ann. of Math. Stud. 200, Princeton Univ. Press, Princeton, NJ, 2019 Zbl 1433.14001 MR 3931681

[18] G. Harder and D. A. Kazhdan, Automorphic forms on $\mathrm{GL}_{2}$ over function fields (after V.G. Drinfel'd). In Automorphic forms, representations and L-functions (Proc. Sympos. Pure Math., Oregon State Univ., Corvallis, Ore., 1977), Part 2, pp. 357-379, Proc. Sympos. Pure Math., XXXIII, Amer. Math. Soc., Providence, R.I., 1979 Zbl 0442.12011 MR 546624

[19] H. Hasse, History of class field theory. In Algebraic Number Theory (Proc. Instructional Conf., Brighton, 1965), pp. 266-279, Thompson, Washington, D.C., 1967 Zbl 0153.07403 MR 0218330

[20] Yu. Hoshi, A. Minamide, and Sh. Mochizuki, Group-theoreticity of numerical invariants and distinguished subgroups of configuration space groups, preprint, 2017. Available from http:// www.kurims.kyoto-u.ac.jp/ motizuki/Numerical\%20invariants\%20and\%20distinguished\% 20subgroups\%20of\%20configuration\%20spaces.pdf

[21] Y. Ihara, On congruence monodromy problems. MSJ Mem. 18, Math. Soc. Japan, Tokyo, 2008 Zbl 0228.14010 MR 2499876

[22] K. I. Ikeda, On the non-abelian global class field theory. Ann. Math. Qué. 37 (2013), no. 2, 129-172 Zbl 1318.11145 MR 3117741

[23] K. I. Ikeda and E. Serbest, Fesenko reciprocity map. Algebra i Analiz 20 (2008), no. 3, 112 162; reprinted in St. Petersburg Math. J. 20 (2009), no. 3, 407-445 Zbl 1206.11138 MR 2454454

[24] K. I. Ikeda and E. Serbest, Generalized Fesenko reciprocity map. Algebra i Analiz 20 (2008), no. 4, 118-159; reprinted in St. Petersburg Math. J. 20 (2009), no. 4, 593-624

Zbl 1206.11140 MR 2473746

[25] K. I. Ikeda and E. Serbest, Non-abelian local reciprocity law. Manuscripta Math. 132 (2010), no. 1-2, 19-49 Zbl 1205.11128 MR 2609287

[26] K. I. Ikeda and E. Serbest, Ramification theory in non-abelian local class field theory. Acta Arith. 144 (2010), no. 4, 373-393 Zbl 1237.11050 MR 2684288

[27] E. Inaba, On matrix equations for Galois extensions of fields with characteristic p. Natur. Sci. Rep. Ochanomizu Univ. 12 (1961), no. 2, 26-36 Zbl 0106.25802 MR 140512

[28] E. Inaba, On generalized Artin-Schreier equations. Natur. Sci. Rep. Ochanomizu Univ. 13 (1962), no. 2, 1-13 Zbl 0115.24903 MR 155817 
[29] K. Iwasawa, A note on functions. In Proceedings of the International Congress of Mathematicians (Cambridge, Mass., 1950). Vol. 1, p. 322, Amer. Math. Soc., Providence, R.I., 1952

[30] K. Iwasawa, Letter to J. Dieudonné. In Zeta functions in geometry (Tokyo, 1990), pp. 445-450, Adv. Stud. Pure Math., 21, Kinokuniya, Tokyo, 1992 Zbl 0835.11002 MR 1210798

[31] K. Iwasawa, Hecke's L-functions. Lectures at Princeton University (Spring 1964). With a foreword by John Coates and Masato Kurihara. SpringerBriefs Math., Springer, Singapore, 2019 Zbl 1455.11007 MR 3969976

[32] K. Kato, A generalization of local class field theory by using $K$-groups. Part I. J. Fac. Sci. Univ. Tokyo Sect. IA Math. 26 (1979), no. 2, 303-376 Zbl 0428.12013 MR 550688; Part II. J. Fac. Sci. Univ. Tokyo Sect. IA Math. 27 (1980), no. 3, 603-683 Zbl 0463.12006 MR 603953

[33] K. Kato, Galois cohomology of complete discrete valuation fields. In Algebraic K-theory, Part II (Oberwolfach, 1980), pp. 215-238, Lecture Notes in Math. 967, Springer, Berlin-New York, 1982 Zbl 0506.12022 MR 689394

[34] K. Kato and S. Saito, Two-dimensional class field theory. In Galois groups and their representations (Nagoya, 1981), pp. 103-152, Adv. Stud. Pure Math. 2, North-Holland, Amsterdam, 1983 Zbl 0544.12011 MR 732466

[35] K. Kato and S. Saito, Global class field theory of arithmetic schemes. In Applications of algebraic K-theory to algebraic geometry and number theory, Part I, II (Boulder, Colo., 1983), pp. 255-331, Contemp. Math. 55, Amer. Math. Soc., Providence, RI, 1986 Zbl 0614.14001 MR 862639

[36] Y. Kawada, Class formations. III. J. Math. Soc. Japan 7 (1955), 453-490 Zbl 0101.02903 MR 79612

[37] M. Kerz, Higher class field theory and the connected component. Manuscripta Math. 135 (2011), no. 1-2, 63-89 Zbl 1221.19004 MR 2783387

[38] M. Kerz and A. Schmidt, Covering data and higher dimensional global class field theory. $J$. Number Theory 129 (2009), no. 10, 2569-2599 Zbl 1187.14028 MR 2541032

[39] N. Kurokawa, Special values of Selberg zeta functions. In Algebraic K-theory and algebraic number theory (Honolulu, HI, 1987), pp. 133-150, Contemp. Math. 83, Amer. Math. Soc., Providence, RI, 1989 Zbl 0684.10038 MR 991979

[40] L. Lafforgue, Chtoucas de Drinfeld et correspondance de Langlands. Invent. Math. 147 (2002), no. 1, 1-241 Zbl 1038.11075 MR 1875184

[41] L. Lafforgue, Le principe de fonctorialité de Langlands comme un problème de généralisation de la loi d'addition, IHES preprint, 2016. Available from http://preprints.ihes.fr/2016/M/ M-16-27.pdf

[42] L. Lafforgue, Du transfert automorphe de Langlands aux formules de Poisson non linéaires. Ann. Inst. Fourier (Grenoble) 66 (2016), no. 3, 899-1012 Zbl 1417.11153 MR 3494164

[43] V. Lafforgue, Chtoucas pour les groupes réductifs et paramétrisation de Langlands globale. J. Amer. Math. Soc. 31 (2018), no. 3, 719-891 Zbl 1395.14017 MR 3787407

[44] S. Lang, Unramified class field theory over function fields in several variables. Ann. of Math. (2) 64 (1956), 285-325 Zbl 0089.26201 MR 83174

[45] S. Lang, Sur les séries $L$ d'une variété algébrique. Bull. Soc. Math. France 84 (1956), 385-407 Zbl 0089.26301 MR 88777

[46] R. P. Langlands, Where stands functoriality today? In Representation theory and automorphic forms (Edinburgh, 1996), pp. 457-471, Proc. Sympos. Pure Math. 61, Amer. Math. Soc., Providence, RI, 1997 Zbl 0901.11032 MR 1476510

[47] S. Lysenko, On the automorphic sheaves for $G S p_{4}, 2019$ arXiv:1901.04447 
[48] S. Lysenko, Moduli of metaplectic bundles on curves and theta-sheaves. Ann. Sci. Éc. Norm. Supér. (4) 39 (2006), no. 3, 415-466 Zbl 1111.14029 MR 2265675

[49] S. Lysenko, Geometric theta-lifting for the dual pair $\mathbb{S O}_{2 m}$, $\mathbb{S p}_{2 n}$. Ann. Sci. Éc. Norm. Supér. (4) 44 (2011), no. 3, 427-493 Zbl 1229.22015 MR 2839456

[50] A. Minamide and H. Nakamura, The automorphism groups of the profinite braid groups. To appear in Amer. J. Math. arXiv:1904.06749

[51] S. Mochizuki, The profinite Grothendieck conjecture for closed hyperbolic curves over number fields. J. Math. Sci. Univ. Tokyo 3 (1996), no. 3, 571-627 Zbl 0889.11020 MR 1432110

[52] S. Mochizuki, The local pro- $p$ anabelian geometry of curves. Invent. Math. 138 (1999), no. 2, 319-423 Zbl 0935.14019 MR 1720187

[53] S. Mochizuki, Topics surrounding the anabelian geometry of hyperbolic curves. In Galois groups and fundamental groups, pp. 119-165, Math. Sci. Res. Inst. Publ., 41, Cambridge Univ. Press, Cambridge, 2003 Zbl 1053.14029 MR 2012215

[54] S. Mochizuki, Topics in absolute anabelian geometry. Part I: Generalities. J. Math. Sci. Univ. Tokyo 19 (2012), no. 2, 139-242 Zbl 1267.14039 MR 2987306; Part II: Decomposition groups and endomorphisms. J. Math. Sci. Univ. Tokyo 20 (2013), no. 2, 171-269

Zbl 1367.14011 MR 3154380; Part III: Global reconstruction algorithms. J. Math. Sci. Univ. Tokyo 22 (2015), no. 4, 939-1156 Zbl 1358.14024 MR 3445958

[55] S. Mochizuki, Alien copies, Gaussians and inter-universal Teichmüller theory. In Interuniversal Teichmüller Theory Summit 2016, pp. 23-192, RIMS Kôkyûroku Bessatsu B84, Res. Inst. Math. Sci. (RIMS), Kyoto, 2021

[56] S. Mochizuki, Inter-universal Teichmüller theory. Part I: Construction of Hodge theaters. Publ. Res. Inst. Math. Sci. 57 (2021), no. 1, 3-207 Zbl 7317908 MR 4225473; Part II: HodgeArakelov-theoretic evaluation. Publ. Res. Inst. Math. Sci. 57 (2021), no. 1, 209-401

Zbl 7317909 MR 4225474; Part III: Canonical splittings of the log-theta-lattice. Publ. Res. Inst. Math. Sci. 57 (2021), no. 1, 403-626 Zbl 7317910 MR 4225475; Part IV: Log-volume computations and set-theoretic foundations. Publ. Res. Inst. Math. Sci. 57 (2021), no. 1, 627723 Zbl 7317911 MR 4225476

[57] F. Morel, $\mathbb{A}^{1}$-algebraic topology over a field. Lecture Notes in Math. 2052, Springer, Heidelberg, 2012 Zbl 1263.14003 MR 2934577

[58] M. Morrow, Integration on product spaces and $\mathrm{GL}_{n}$ of a valuation field over a local field. Commun. Number Theory Phys. 2 (2008), no. 3, 563-592 Zbl 1171.28303 MR 2482943

[59] M. Morrow, Integration on valuation fields over local fields. Tokyo J. Math. 33 (2010), no. 1, 235-281 Zbl 1203.11081 MR 2682892

[60] J. Neukirch, Algebraic number theory. Grundlehren Math. Wiss. 322, Springer, Berlin, 1999 Zbl 0956.11021 MR 1697859

[61] J. Neukirch, Class field theory. Grundlehren Math. Wiss. 280, Springer, Berlin, 1986 Zbl 0587.12001 MR 819231

[62] A. N. Parshin, Local class field theory. Proc. Steklov Inst. Math. 165 (1985), no. 3, 157-185 Zbl 0579.12012 MR 752939

[63] A. N. Parshin, Galois cohomology and the Brauer group of local fields. Proc. Steklov Inst. Math. (1991), no. 4, 191-201 Zbl 0731.11064 MR 1092028

[64] N. Schappacher, On the history of Hilbert's twelfth problem: a comedy of errors. In Matériaux pour l'histoire des mathématiques au XX $X^{\mathrm{e}}$ siècle (Nice, 1996), pp. 243-273, Sémin. Congr. 3, Soc. Math. France, Paris, 1998 Zbl 1044.01530 MR 1640262

[65] M. Suzuki, Positivity of certain functions associated with analysis on elliptic surfaces. J. Number Theory 131 (2011), no. 10, 1770-1796 Zbl 1237.11028 MR 2811546 
[66] M. Suzuki, Two-dimensional adelic analysis and cuspidal automorphic representations of GL(2). In Multiple Dirichlet series, L-functions and automorphic forms, pp. 339-361, Progr. Math. 300, Birkhäuser/Springer, New York, 2012 Zbl 1277.11074 MR 2952583

[67] A. Tamagawa, The Grothendieck conjecture for affine curves. Compositio Math. 109 (1997), no. 2, 135-194 Zbl 0899.14007 MR 1478817

[68] J. T. Tate, Fourier analysis in number fields, and Hecke's zeta-functions. In Algebraic Number Theory (Proc. Instructional Conf., Brighton, 1965), pp. 305-347, Thompson, Washington, D.C., 1967 MR 0217026

[69] S. Tsujimura, Combinatorial Belyi cuspidalization and arithmetic subquotients of the Grothendieck-Teichmüller group. Publ. Res. Inst. Math. Sci. 56 (2020), no. 4, 779-829 Zbl 1460.14068 MR 4162297

[70] S. V. Vostokov, Explicit formulas for the Hilbert symbol. In Invitation to higher local fields (Münster, 1999), pp. 81-90, Geometry and Topology Monographs 3, Geometry and Topology Publications, Coventry, 2000. Available from https://msp.org/gtm/2000/03/p008.xhtml

[71] R. Waller, Measure and integration on $\mathrm{GL}_{2}$ over a two-dimensional local field. New York J. Math. 25 (2019), 396-422 Zbl 1457.11167 MR 3982247

[72] A. Weil, Basic number theory. Russian edn, Osnovy teorii chisel, Mir, 1972

[73] G. Wiesend, A construction of covers of arithmetic schemes. J. Number Theory 121 (2006), no. 1, 118-131 Zbl 1120.14014 MR 2268759

[74] G. Wiesend, Class field theory for arithmetic schemes. Math. Z. 256 (2007), no. 4, 717-729 Zbl 1115.14016 MR 2308885

Received 5 May 2021.

\section{Ivan Fesenko}

School of Mathematical Sciences, University of Nottingham, Nottingham NG7 2RD, UK;

ivan.b.fesenko@gmail.com 